\title{
Competing orders, competing anisotropies, and multicriticality: The case of Co-doped $\mathrm{YbRh}_{2} \mathrm{Si}_{2}$
}

\author{
Eric C. Andrade, ${ }^{1,2}$ Manuel Brando, ${ }^{3}$ Christoph Geibel, ${ }^{3}$ and Matthias Vojta ${ }^{1}$ \\ ${ }^{1}$ Institut für Theoretische Physik, Technische Universität Dresden, 01062 Dresden, Germany \\ ${ }^{2}$ Instituto de Física Teórica, Universidade Estadual Paulista, Rua Dr. Bento Teobaldo Ferraz, 271 - Bl. II, 01140-070, São Paulo, SP, Brazil \\ ${ }^{3}$ Max-Planck-Institut für Chemische Physik fester Stoffe, Nöthnitzer Straße 40, 01187 Dresden, Germany \\ (Received 20 December 2013; revised manuscript received 11 August 2014; published 22 August 2014)
}

\begin{abstract}
Motivated by the unusual evolution of magnetic phases in stoichiometric and Co-doped $\mathrm{YbRh}_{2} \mathrm{Si}_{2}$, we study Heisenberg models with competing ferromagnetic and antiferromagnetic ordering combined with competing anisotropies in exchange interactions and $g$ factors. Utilizing large-scale classical Monte Carlo simulations, we analyze the ingredients required to obtain the characteristic crossing point of uniform susceptibilities observed experimentally near the ferromagnetic ordering of $\mathrm{Yb}\left(\mathrm{Rh}_{0.73} \mathrm{Co}_{0.27}\right)_{2} \mathrm{Si}_{2}$. The models possess multicritical points, which we speculate to be relevant for the behavior of clean as well as doped $\mathrm{YbRh}_{2} \mathrm{Si}_{2}$. We also make contact with experimental data on $\mathrm{YbNi}_{4} \mathrm{P}_{2}$, where a similar susceptibility crossing has been observed.
\end{abstract}

DOI: 10.1103/PhysRevB.90.075138 PACS number(s): 64.60.Kw, 71.27.+a, 75.10.Hk, 75.30.Gw

\section{INTRODUCTION}

Magnetic quantum phase transitions (QPTs) in solids are an active area of research, with many unsolved problems present particularly for metallic systems [1,2]. While the simplest theoretical models assume a single type of ordering instability and $\mathrm{SU}(2)$ spin rotation symmetry, real materials often possess additional ingredients which complicate the interpretation of experimental data: (i) a competition of multiple ordered states, e.g., with different ordering wave vectors and/or different spin structures that may result from geometric frustration or multiple nesting conditions, and (ii) magnetic anisotropies that can modify ordered states and induce additional crossover energy scales.

The tetragonal heavy-fermion metal $\mathrm{YbRh}_{2} \mathrm{Si}_{2}$ is a prominent example for anisotropic and competing magnetic orders. Its uniform magnetic susceptibility is an order of magnitude larger for fields $B_{a b}$ applied in the basal plane as compared to fields $B_{c}$ along the $c$ axis. Stoichiometric $\mathrm{YbRh}_{2} \mathrm{Si}_{2}$ displays an ordered phase below $T_{\mathrm{N}}=70 \mathrm{mK}$, believed to be an antiferromagnet with moments oriented in the basal plane. This order is destroyed at a field-driven QPT at $B_{a b}^{\text {crit }}=60 \mathrm{mT}$; the corresponding $c$-axis critical field is $B_{c}^{\text {crit }}=0.66 \mathrm{~T}[3,4]$.

Upon applying hydrostatic pressure or doping with Co, both $T_{\mathrm{N}}$ and $B^{\text {crit }}$ increase which is primarily caused by a reduction of Kondo screening. Moreover, a second phase transition at $T_{\mathrm{L}}<T_{\mathrm{N}}$ occurs at small fields, Fig. 1(a), with the precise nature of the phase for $T<T_{\mathrm{L}}$ being unknown $[5,6]$. Interestingly, an almost divergent uniform susceptibility was reported for stoichiometric $\mathrm{YbRh}_{2} \mathrm{Si}_{2}$, hinting at the presence of ferromagnetic correlations [7]. However, in-plane ferromagnetic order could be detected neither under pressure nor with doping.

Given this state of affairs, it came as a surprise that $\mathrm{Yb}\left(\mathrm{Rh}_{0.73} \mathrm{Co}_{0.27}\right)_{2} \mathrm{Si}_{2}$ was recently found [8] to develop ferromagnetic order below $T_{\mathrm{C}}=1.3 \mathrm{~K}$ with moments oriented along the $c$ axis, which was identified as the hard magnetic axis from all previous measurements. This "switch" of response anisotropies in $\mathrm{Yb}\left(\mathrm{Rh}_{0.73} \mathrm{Co}_{0.27}\right)_{2} \mathrm{Si}_{2}$ is reflected in the susceptibilities, where $\chi_{a b}(T)$ and $\chi_{c}(T)$ display a characteristic crossing point at $T_{\mathrm{X}}$ slightly above $T_{\mathrm{C}}$ [Fig. 1(b)]. Furthermore, the evolution of $T_{\mathrm{L}}$ with doping in
$\mathrm{Yb}\left(\mathrm{Rh}_{1-x} \mathrm{Co}_{x}\right)_{2} \mathrm{Si}_{2}$ [Fig. 1(a)] suggests that the transition at $T_{\mathrm{L}}$ involves a ferromagnetic ordering component for $x<0.27$. Parenthetically, we note that antiferromagnetism takes over again for $x \gtrsim 0.6$, with $\mathrm{YbCo}_{2} \mathrm{Si}_{2}$ displaying a Néel transition at $T_{\mathrm{N}}=1.65 \mathrm{~K}$ and a large in-plane ordered moment of $1.4 \mu_{B}[6,9]$.

Taken together, the data show that the magnetism of $\mathrm{Yb}\left(\mathrm{Rh}_{1-x} \mathrm{Co}_{x}\right)_{2} \mathrm{Si}_{2}$ is determined by competing antiferromagnetic (AFM) and ferromagnetic (FM) orders, which, moreover, are characterized by competing anisotropies.

In this paper we propose that Heisenberg models of local moments can account for many of the unusual magnetic properties of $\mathrm{Yb}\left(\mathrm{Rh}_{1-x} \mathrm{Co}_{x}\right)_{2} \mathrm{Si}_{2}$ by invoking multiple magnetic interactions and a competition between anisotropic exchange and anisotropic $g$ factors. We primarily focus on the behavior of $\mathrm{Yb}\left(\mathrm{Rh}_{0.73} \mathrm{Co}_{0.27}\right)_{2} \mathrm{Si}_{2}$ in the vicinity of its finite-temperature phase transition which can be modeled classically. For the simplest models, we investigate thermodynamic properties using large-scale Monte Carlo (MC) simulations and provide an explicit comparison to experimental susceptibility data. Based on our results, we propose that multicritical points, naturally present in our modeling, are of relevance for understanding the unusual quantum criticality in stoichiometric $\mathrm{YbRh}_{2} \mathrm{Si}_{2}$. Finally, we also discuss the behavior of the heavy-fermion ferromagnet $\mathrm{YbNi}_{4} \mathrm{P}_{2}$, which displays a similar "switch" of magnetic response anisotropy [10,11].

The body of the paper is organized as follows: In Sec. II we describe the family of anisotropic Heisenberg models to be studied, together with methodological aspects of the MC simulations. Section III is devoted to a detailed modeling of $\mathrm{Yb}\left(\mathrm{Rh}_{0.73} \mathrm{Co}_{0.27}\right)_{2} \mathrm{Si}_{2}$. It starts with a discussion of competing magnetic anisotropies and then introduces competing FM and AFM ordering tendencies, in order to match experimental observations. In Sec. IV we then discuss the presence of multicritical points in the phase diagrams of our models and speculate on the role of quantum bicriticality in the phase diagram of $\mathrm{YbRh}_{2} \mathrm{Si}_{2}$. Finally, in Sec. $\mathrm{V}$ we briefly discuss the case of $\mathrm{YbNi}_{4} \mathrm{P}_{2}$. A short summary concludes the paper. In the Appendix we illustrate the effects of an additional single-ion anisotropy, not present in the spin- $1 / 2$ models discussed in the bulk of the paper. 

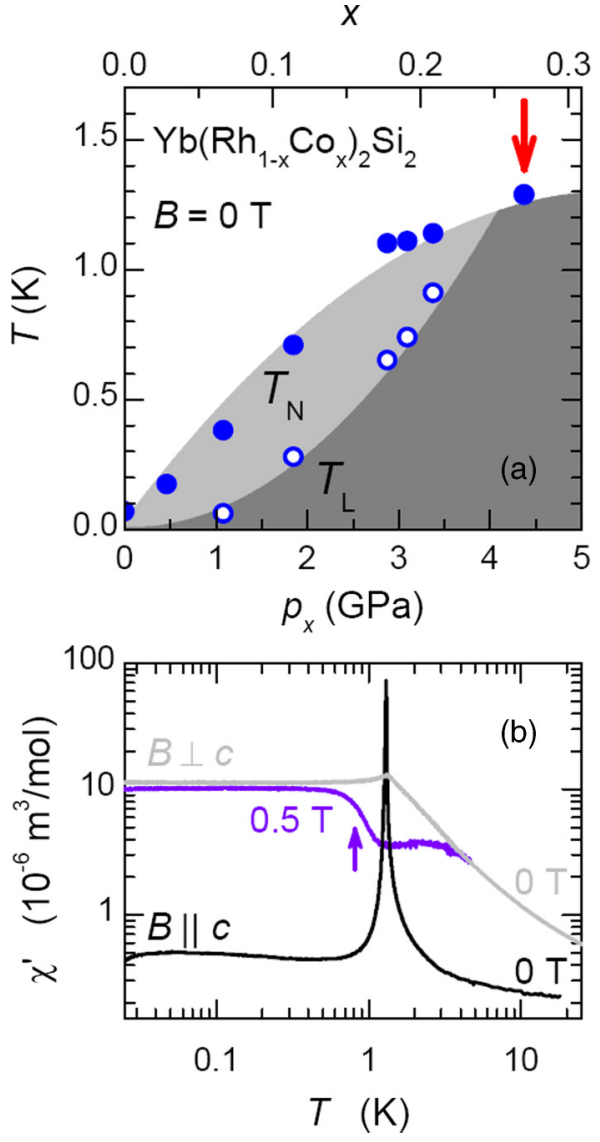

FIG. 1. (Color online) (a) Temperature-composition phase diagram of $\mathrm{YbRh}_{2} \mathrm{Si}_{2}$ showing the two magnetic transitions at $T_{\mathrm{N}}$ and $T_{\mathrm{L}}$. Pressure (lower axis) has an effect similar to Co doping (upper axis). (We note that ongoing studies suggest an even more complex phase diagram with canted AFM order below $T_{\mathrm{L}}$ for $x<0.2$.) (b) Susceptibility data from $\mathrm{Yb}\left(\mathrm{Rh}_{0.73} \mathrm{Co}_{0.27}\right)_{2} \mathrm{Si}_{2}$ showing the switch of the anisotropy of the magnetic response as function of temperature. Figure reproduced from Ref. [8].

\section{MODEL AND SIMULATIONS}

\section{A. Model and magnetic anisotropies}

The $\mathrm{Yb}^{3+}$ ions in $\mathrm{YbRh}_{2} \mathrm{Si}_{2}$ are in a $4 f^{13}$ configuration in a tetragonal crystal field. The ionic ground state is a $\Gamma^{7}$ Kramers doublet [12], which can be represented by an effective (pseudo)spin-1/2 moment, which further couples to conduction electrons via a Kondo exchange coupling. Due to strong spin-orbital coupling, $\mathrm{SU}(2)$ spin symmetry is broken and magnetic anisotropies appear: (i) The (pseudo)spins couple to an external Zeeman field in an anisotropic fashion, leading to an anisotropic $g$ tensor. (ii) Magnetic interactions between the moments will be anisotropic in spin space, which applies to both direct exchange and Ruderman-KittelKasuya-Yosida (RKKY) interactions. A single-ion anisotropy is forbidden for elementary spin-1/2; for completeness we discuss its effects in models of spins $S \geqslant 1$ in the Appendix.

In this paper, we consider spin-only models of $\mathrm{Yb}$ moments in $\mathrm{YbRh}_{2} \mathrm{Si}_{2}$. These models will be of Heisenberg type; to account for competing phases, we will include longer-range exchange interactions. For simplicity, we place the moments on a cubic lattice and consider models of the form

$$
\mathcal{H}=-\sum_{\langle i, j\rangle \alpha} J_{1}^{\alpha} S_{i}^{\alpha} S_{j}^{\alpha}-\sum_{\langle\langle i, j\rangle\rangle \alpha} J_{2}^{\alpha} S_{i}^{\alpha} S_{j}^{\alpha}-\sum_{i \alpha} g_{\alpha} h_{\alpha} S_{i}^{\alpha}
$$

where $J_{1}$ and $J_{2}$ denote couplings to first and second neighbors. $S_{i}^{\alpha}$ is the $\alpha=x, y, z$ component of the moment at site $i, h_{\alpha}$ represents the external magnetic field, and $g_{\alpha}$ are the (diagonal) components of the $g$ tensor. Because the $\mathrm{Yb}$ moments are in a tetragonal crystal field, we have $g_{x}=g_{y} \equiv g_{a b}$ and $g_{z} \equiv g_{c}$. Analogously, the exchange interaction between the $\mathrm{Yb}^{3+}$ ions can be parameterized as $J_{1}^{x}=J_{1}^{y}=J_{1}\left(1+\Delta_{1}\right)$ and $J_{1}^{z} \equiv$ $J_{1}$. For simplicity, we consider isotropic second-neighbor couplings, with $J_{2}^{\alpha}=J_{2}$. Being interested in the behavior near the finite-temperature phase transition, we approximate the quantum spins as classical vectors with $\left|\vec{S}_{i}\right|=1$.

\section{B. Monte Carlo simulations}

To access the finite-temperature behavior of Eq. (1) we perform equilibrium MC simulations on cubic lattices of size $N=L \times L \times L$, with $L \leqslant 32$ and periodic boundary conditions. We employ the single-site Metropolis algorithm combined with microcanonical steps to improve the sampling at lower temperatures. Typically, we run $10^{6} \mathrm{MC}$ steps (MCS) as initial thermalization followed by $10^{6} \mathrm{MCS}$ to obtain thermal averages, which are calculated by dividing the measurement steps into 10 bins. Here one MCS corresponds to one attempted spin flip per site. We consider the same number of Metropolis and microcanonical steps. In all our results we set $k_{B}=1$ and $a=1$, where $k_{B}$ is the Boltzmann constant and $a$ is the lattice spacing and use $J_{1}$ as our energy scale.

From the MC data, we calculate thermodynamic observables as the specific heat $C$ and the uniform magnetic susceptibilities, both along the $c$ axis,

$$
\chi_{c}(T)=g_{c}^{2} \frac{N}{T}\left(\left\langle m_{c}^{2}\right\rangle-\left\langle m_{c}\right\rangle^{2}\right),
$$

and in the $a b$ plane,

$$
\chi_{a b}(T)=\frac{1}{2} g_{a b}^{2} \frac{N}{T}\left\langle m_{a b}^{2}\right\rangle,
$$

where $\langle\cdots\rangle$ denotes the MC average, $m_{c}=M_{z}$, and $m_{a b}=$ $\left(M_{x}^{2}+M_{y}^{2}\right)^{1 / 2}$ with $M_{\alpha}=N^{-1} \sum_{i} S_{i}^{\alpha}$. Due to its definition, $m_{a b}$ incorporates the fact that the $x$ and $y$ components of $\vec{S}$ are equivalent. In $\chi_{a b}$ no subtraction of expectation values is necessary, as we will only consider ferromagnetic states with order along the $c$ axis. In the linear-response limit, $g$ factors do not enter the MC simulations directly but appear as prefactors in Eqs. (2) and (3) only.

Further, we analyze phase transitions via the Binder cumulant [13] and we characterize ordered states by the static 

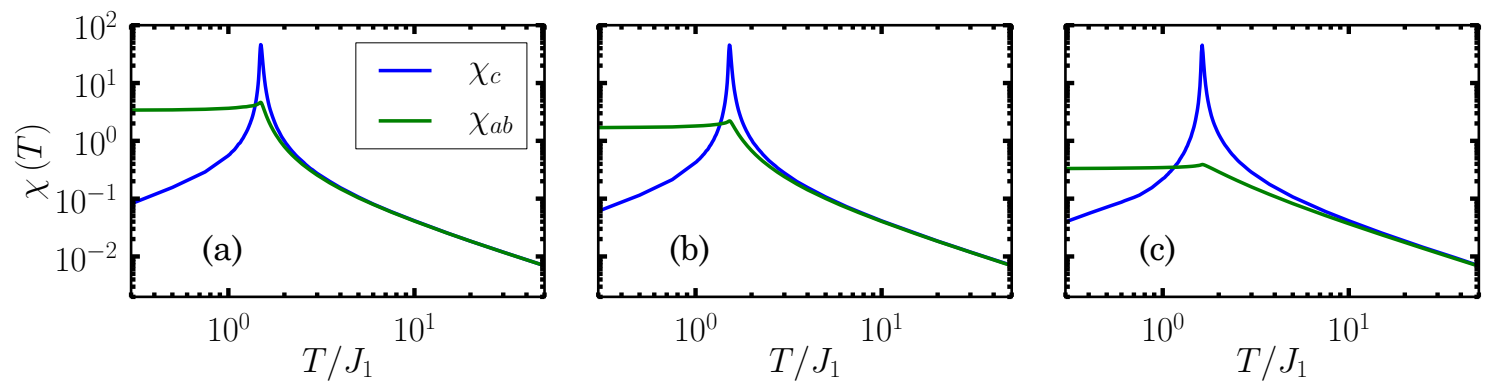

FIG. 2. (Color online) Anisotropic susceptibilities $\chi_{a b}$ (green) and $\chi_{c}$ (blue) on a log-log scale for FM $\left(J^{x, y}>0\right)$ spin exchange in the $a b$ plane, $J_{2}=0, g_{a b}=g_{c}$, and $L=16$ : (a) $\Delta=-0.05$; (b) $\Delta=-0.10$; and (c) $\Delta=-0.50$. Finite-size effects are negligible except for temperatures within $0.2 \%$ of $T_{\mathrm{C}}$.

spin structure factors:

$$
\begin{gathered}
S_{c}(\vec{q})=\frac{1}{N} \sum_{i, j} e^{-i \vec{q} \cdot \vec{r}_{i j}}\left\langle S_{i}^{z} S_{j}^{z}\right\rangle, \\
S_{a b}(\vec{q})=\frac{1}{N} \sum_{i, j} e^{-i \vec{q} \cdot \vec{r}_{i j}}\left\langle S_{i}^{x} S_{j}^{x}+S_{i}^{y} S_{j}^{y}\right\rangle .
\end{gathered}
$$

In a long-range ordered phase $S_{c(a b)}(\vec{q}) / N \rightarrow m_{c(a b)}^{2} \delta_{\vec{q}, \vec{Q}}$, where $\delta$ is the Kronecker delta and $\vec{Q}$ is the ordering wave vector. Note that $m_{c(a b)} \rightarrow 1$ as $T \rightarrow 0$, because the classical ground state is fluctuationless. The behavior of $S_{c(a b)}(\vec{q})$ near $\vec{Q}$ allows one to extract the correlation length $\xi_{c(a b)}(T)$ characterizing the magnetic order. In our finite-size simulations, the ordering temperature for the second-order phase transition $T_{\mathrm{C}}$ is efficiently extracted from the crossing points of $\xi(T) / L$ data for different $L$, according to the scaling law $\xi(T) / L=f\left[L^{1 / v}\left(T-T_{\mathrm{C}}\right)\right]$, where $f(x)$ is a scaling function and $v$ is the correlation length exponent.

\section{MODELING OF $\mathrm{Yb}\left(\mathrm{Rh}_{0.73} \mathrm{Co}_{0.27}\right)_{2} \mathrm{Si}_{2}$}

In this section, we describe a stepwise modeling of magnetism in $\mathrm{Yb}\left(\mathrm{Rh}_{0.73} \mathrm{Co}_{0.27}\right)_{2} \mathrm{Si}_{2}$ using spin models of the form (1), with the goal of reproducing the temperature dependence of $\chi(T)$ in Fig. 1(b). Conduction electrons are not part of this modeling, i.e., we assume that the magnetic properties can be described in terms of (effective) local-moment models. We note that this assumption is not in contradiction with possible Kondo screening of the $\mathrm{Yb} 4 f$ moments: First, in Kondo-lattice systems the local-moment contributions to the magnetic response are typically much larger than those of the conduction electrons. Second, universality dictates that, even in cases where magnetism is fully itinerant, local-moment models successfully describe many long-wavelength magnetic properties [14].

\section{A. Competing anisotropies and bicriticality}

A central observation in the phase diagram [6,8] of $\mathrm{Yb}\left(\mathrm{Rh}_{1-x} \mathrm{Co}_{x}\right)_{2} \mathrm{Si}_{2}$ [Fig. 1(a)] is that the two thermal phase transitions at $T_{\mathrm{N}}$ and $T_{\mathrm{L}}$, existing for small $x$, merge close to $x=0.27$. Combining the facts that $T_{\mathrm{N}}$ marks a transition into an in-plane antiferromagnet and the state below $T_{\mathrm{C}}$ at $x=0.27$ is a $c$-axis ferromagnet suggests the competition of these two instabilities, with a proximate bicritical (or, more generally, multicritical) point.
We start by focusing on the competition between in-plane and $c$-axis order, which can be captured, in a spin-1/2 model as in Eq. (1), by anisotropic exchange couplings, with $\left|J_{1}^{x, y}\right|=\left|J_{1}^{z}\right|\left(1+\Delta_{1}\right)$. Degeneracy of the two orders is trivially reached at the Heisenberg point $\Delta_{1}=0$, where the transition corresponds to a bicritical point. A transition to $c$-axis order as in $\mathrm{Yb}\left(\mathrm{Rh}_{0.73} \mathrm{Co}_{0.27}\right)_{2} \mathrm{Si}_{2}$ requires $\Delta_{1}<0$. Sample susceptibilities results assuming FM $\left(J^{x, y}>0\right)$ spin exchange in the $a b$ plane are shown in Fig. 2, where we see that while $\chi_{c}(T)$ is essentially independent of the anisotropy $\Delta_{1}$, $\chi_{a b}(T)$ is considerably enhanced as we approach the bicritical point, due to the increase in the in-plane fluctuations. The behavior of the magnetic susceptibilities in the vicinity of a bicritical point is further explored in Appendix A.

The fact that, experimentally, $\chi_{c} \ll \chi_{a b}$ for a large range of temperatures above the transition (essentially from $T_{\mathrm{C}}$ up to $100 \mathrm{~K}$ ) implies $g_{c}<g_{a b}$, i.e., the $g$-factor anisotropy is opposite to the exchange anisotropy. Indeed, for undoped $\mathrm{YbRh}_{2} \mathrm{Si}_{2}$ a strong $g$-factor anisotropy of $g_{a b} / g_{c} \approx 20$ has been deduced from electron-spin-resonance (ESR) experiments [12]. For $\mathrm{Yb}\left(\mathrm{Rh}_{0.73} \mathrm{Co}_{0.27}\right)_{2} \mathrm{Si}_{2}$ we have [8] $g_{a b} / g_{c}=6.4$, and we note that the end member of the doping series, $\mathrm{YbCo}_{2} \mathrm{Si}_{2}$, has a smaller anisotropy, $g_{a b} / g_{c} \approx 2.5$, indicating a considerable variation of the $g$-factor anisotropy ratio throughout the series, even though no change in the symmetry of the ground-state doublet is observed $[9,15]$.

In a modeling of the susceptibility data of $\mathrm{Yb}\left(\mathrm{Rh}_{0.73} \mathrm{Co}_{0.27}\right)_{2} \mathrm{Si}_{2}$ under the assumption of exclusively nearest-neighbor ferromagnetic coupling $J_{1}^{\alpha}>0$ (this assumption will be relaxed in Sec. III B below) we are left with the following free parameters: the overall energy scale $J_{1}$ which determines $T_{\mathrm{C}}$, the exchange anisotropy $\Delta$, and the $g$-factor anisotropy $g_{a b} / g_{c}$. (The absolute value of $\chi$, or $g$, is simply adjusted to the data by matching the low- $T$ value of $\chi_{a b}$.) To determine $\Delta$ and $g_{a b} / g_{c}$ different strategies appear possible; we found the following useful: First, $\Delta$ is chosen to match the temperature dependence of the nondivergent $\chi_{a b}(T)$ near $T_{\mathrm{C}}$ (where $\chi_{c}$ diverges). In general, small $\Delta$ should be chosen to ensure proximity to bicriticality. Second, with $\Delta$ fixed, the crossing temperature $T_{\mathrm{X}}$ of $\chi_{a b}$ and $\chi_{c}$ can be used to determine the $g$-factor anisotropy by demanding that experiment and theory yield the same $T_{\mathrm{X}} / T_{\mathrm{C}}$ ratio; experimentally this number is $T_{\mathrm{X}} / T_{\mathrm{C}}=1.016$. After this procedure is implemented, we add a constant susceptibility $\chi_{v}$ to mimic the Van Vleck contribution arising from higher 

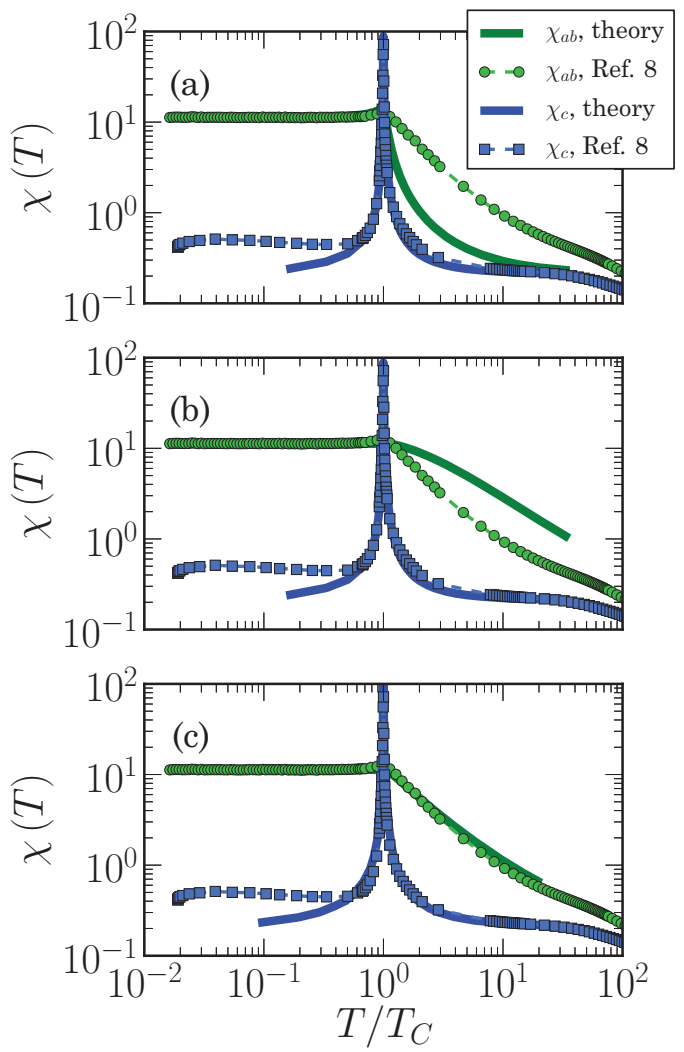

FIG. 3. (Color online) Comparison between the numerical and experimental susceptibilities $\chi(T)$ as a function of $T / T_{\mathrm{C}}$ on a $\log -\log$ scale. The continuous curves show the simulation results, $\chi_{a b}$ in green and $\chi_{c}$ in blue, obtained for $\Delta=-0.05$ and $L=32$ in Eq. (1), with different exchange parameters in panels (a)-(c). The dashed lines with symbols represent the corresponding experimental susceptibilities reported in Ref. [8] in units of $10^{-6} \mathrm{~m}^{3} / \mathrm{mol}$, with $T_{\mathrm{C}}=1.30 \mathrm{~K}$. (a) FM $\left(J^{x, y}>0\right)$ spin exchange in the $a b$ plane, with $g_{a b} / g_{c}=$ 2.5 and $J_{1}=0.87 \mathrm{~K}$. (b) Same as (a), but for AFM $\left(J^{x, y}<0\right)$ spin exchange in the $a b$ plane and $g_{a b} / g_{c}=16.4$. (c) Same as (b), with additional isotropic next-nearest-neighbor FM coupling $J_{2}$, with $J_{2}=$ $J_{1}, J_{1}=0.25 \mathrm{~K}$, and $g_{a b} / g_{c}=7.3$. In all simulation results, we have added a constant Van Vleck background $\chi_{v}=0.20 \times 10^{-6} \mathrm{~m}^{3} / \mathrm{mol}$, as is seen in $\mathrm{Yb}\left(\mathrm{Rh}_{0.73} \mathrm{Co}_{0.27}\right)_{2} \mathrm{Si}_{2}$ [8].

crystal-electric-field levels which are not part of our model. The value chosen is $\chi_{v}=0.20 \times 10^{-6} \mathrm{~m}^{3} / \mathrm{mol}$, which is close to the value of $0.172 \times 10^{-6} \mathrm{~m}^{3} / \mathrm{mol}$ deduced for $\mathrm{Yb}\left(\mathrm{Rh}_{0.73} \mathrm{Co}_{0.27}\right)_{2} \mathrm{Si}_{2}$ [8].

For exclusively nearest-neighbor ferromagnetic coupling, the first step cannot be satisfactorily followed: The temperature variation of $\chi_{a b}$ is too large compared to experiment. A sample set of data is presented in Fig. 3(a). Here $\Delta=-0.05$ has been chosen as a compromise; fixing $T_{\mathrm{X}} / T_{\mathrm{C}}$ then yields $g_{a b} / g_{c}=2.5$. The poor agreement with experiment will be cured upon considering dominant in-plane antiferromagnetic correlations below.

\section{B. Competing ferromagnetism and antiferromagnetism}

While the significant increase of $\chi_{a b}$ towards low temperatures in both Co-doped and undoped $\mathrm{YbRh}_{2} \mathrm{Si}_{2}$ reflects the presence of ferromagnetic exchange, the tendency towards antiferromagnetism cannot be ignored. Since undoped $\mathrm{YbRh}_{2} \mathrm{Si}_{2}$ is believed (and $\mathrm{YbCo}_{2} \mathrm{Si}_{2}$ was proved) to display low-temperature antiferromagnetic order, it is very likely that strong in-plane antiferromagnetism exist in $\mathrm{Yb}\left(\mathrm{Rh}_{0.73} \mathrm{Co}_{0.27}\right)_{2} \mathrm{Si}_{2}$ as well.

First we repeat the analysis described in Sec. III A with purely AFM in-plane exchange, i.e., we consider the model (1) with $J_{1}^{x, y}=-J_{1}\left(1+\Delta_{1}\right)<0$ and $J_{1}^{z}=J_{1}>0$. Corresponding results are presented in Fig. 3(b). Since the coupling of the spins' $z$ components remains FM, $\chi_{c}$ does not change as compared to Fig. 3(a). In contrast, $\chi_{a b}$ is not only strongly suppressed but also shows much less temperature variation, as expected for an antiferromagnet. For $T>T_{\mathrm{C}}$ the susceptibility follows roughly $\chi_{a b} \propto 1 /\left(T-\Theta_{a b}\right)$ with a Curie-Weiss temperature $\Theta_{a b} \sim J^{x, y}<0$, which results in a flattening towards low temperature. To restore the $\chi$ crossing at the experimental value of $T_{\mathrm{X}} / T_{\mathrm{C}}$, we now need to assume a $g$ factor ratio of $g_{a b} / g_{c}=16.4$. The behavior of $\chi_{a b}$ for $T<T_{\mathrm{C}}$ is now much closer to that observed in $\mathrm{Yb}\left(\mathrm{Rh}_{0.73} \mathrm{Co}_{0.27}\right)_{2} \mathrm{Si}_{2}$. However, its very slow decay upon heating above $T_{\mathrm{C}}$ still indicates a rather poor match with the experiment.

The natural conclusion is that both FM and AFM tendencies are present for the in-plane order, a qualitative conclusion reached earlier on the basis of experimental data [7]. There are various ways to implement the competition of FM and AFM order in a microscopic spin model. Here we choose a simple and frustration-free way. We employ an isotropic ferromagnetic second-neighbor coupling $J_{2}$. For $J_{1}^{x y}=0$ and $J_{2} \neq 0$, we have two decoupled sublattices which independently order ferromagnetically. As first discussed in Ref. [16], thermal fluctuations select collinear states such that the wave vectors $\vec{Q}=(0,0,0)$ (sublattices parallel) and $\vec{Q}=(\pi, \pi, \pi)$ (sublattices antiparallel) are degenerated. A finite $J_{1}^{x y} \gtrless 0$ can then be used to tip the balance between dominant ferromagnetic and antiferromagnetic in-plane correlations.

Results with $J_{2}$ included are presented in Fig. 3(c). As anticipated, $J_{2}^{x y}>0$ enhances the FM fluctuations in the $a b$ plane, which has two immediate effects: (i) The $g_{a b} / g_{c}$ ratio required to fit $T_{\mathrm{X}} / T_{\mathrm{C}}$ decreases with increasing $J_{2}$ and (ii) the behavior of $\chi_{a b}$ for $T>T_{\mathrm{C}}$ also approaches the one from $\chi_{a b}^{\exp }$ as we increase $J_{2}$, indicating that the effects of this extra exchange term are considerably felt for $T \gtrsim T_{\mathrm{C}}$. [For $J_{2}>$ $0.5 J_{1}(1+\Delta)$ we have a Curie-Weiss temperature $\Theta_{a b}>0$.]

Whereas for FM in-plane correlation $\chi_{a b}(T)$ shows a distinct dependence on the anisotropy $\Delta_{1}$ (Fig. 2), this effect is considerably weaker for AFM correlations since in this case $\chi_{a b}$ is itself strongly suppressed. Therefore, we obtain similar results, as in Fig. 3(c), for $\Delta_{1}=-0.10$ and even $\Delta_{1}=-0.50$. While this diminishes our predictive power with respect to the precise value of the anisotropy, we recall that Fig. 1(b) suggests proximity to the bicritical point, which constrains $\Delta_{1}$ to be small.

The good match of theoretical and experimental susceptibilities in Fig. 3(c) suggests that our classical anisotropic Heisenberg model captures the essentials of the ferromagnetic ordering process in $\mathrm{Yb}\left(\mathrm{Rh}_{0.73} \mathrm{Co}_{0.27}\right)_{2} \mathrm{Si}_{2}$.

We note that the scenario of different competing kinds of in-plane exchange interactions is also (indirectly) supported by the complicated propagation vectors observed in pure $\mathrm{YbCo}_{2} \mathrm{Si}_{2}$, where $\vec{Q}=2 \pi(1 / 4,0.08,1)$ and 
$\vec{Q}=2 \pi(1 / 4,1 / 4,1)$ for the high- $T$ and low- $T$ phases, respectively. While such $\vec{Q}$ might also result from competition of purely AFM exchanges, our analysis of the $T$ dependence of $\chi_{a b}$ for $T>T_{\mathrm{C}}$ gives a very strong hint that some of these in-plane exchanges have to be ferromagnetic.

\section{MULTICRITICALITY: CLASSICAL AND QUANTUM}

The modeling of $\mathrm{Yb}\left(\mathrm{Rh}_{0.73} \mathrm{Co}_{0.27}\right)_{2} \mathrm{Si}_{2}$ presented so far suggests the relevance of bicritical behavior, arising from the competition of in-plane antiferromagnetism and out-ofplane ferromagnetism. Indeed, the temperature-doping phase diagram of $\mathrm{YbRh}_{2} \mathrm{Si}_{2}$ (Fig. 1) indicates the presence of a finitetemperature bicritical point at a doping level of $x \approx 25 \%$. In the simplest scenario, the lower-temperature phase transition line emerging from this point, labeled $T_{\mathrm{L}}$, would then correspond to a first-order transition between antiferromagnetic and ferromagnetic phases, but more complicated structures with mixed (e.g., canted) orders are possible as well (those are not described by the simple Heisenberg models of Sec. II).

A remarkable feature of the $T_{\mathrm{N}}$ and $T_{\mathrm{L}}$ lines in Fig. 1 is that $T_{\mathrm{L}}$, although more strongly suppressed with decreasing $x$ as compared to $T_{\mathrm{N}}$, appears to be finite almost down to $x=0$. Given that $T_{\mathrm{N}}$ is almost vanishing there as well, this invites speculations about (approximate) quantum bicriticality near $x=0$. This scenario would involve simultaneous quantum criticality of both itinerant in-plane antiferromagnetism and $c$-axis ferromagnetism. In fact, some observations in stoichiometric (or slightly Ge-doped) $\mathrm{YbRh}_{2} \mathrm{Si}_{2}$ have been suggested to be compatible with ferromagnetic quantum criticality. This includes the $T^{-0.7}$ dependence of the Grüneisen parameter $\Gamma$ and the $T^{-0.3}$ dependence of the specific heat $C / T$ at low temperatures above $T_{\mathrm{N}}$ (see Ref. [2]). However, this agreement has mainly been considered fortuitous, as the ordered phase of stoichiometric $\mathrm{YbRh}_{2} \mathrm{Si}_{2}$ is not ferromagnetic. Quantum bicriticality would thus provide a new angle on the observations.

On the theoretical side, we note that bicriticality involving in-plane and $c$-axis order by itself is not exotic but simply corresponds to a point with an emergent higher symmetry, here $\mathrm{O}(3)$. What is, however, interesting and potentially exotic is quantum bicriticality involving itinerant antiferromagnetism and ferromagnetism, which has not been studied theoretically. Within the Landau-Ginzburg-Wilson description, pioneered by Hertz [17], these two transitions have different dynamical exponents, $z=2$ and 3 , respectively, such that one can expect rich and nontrivial crossover phenomena, even if both transitions are above their respective upper-critical dimension [18]. This defines a fascinating field for future theoretical studies [19].

We note that another possible candidate for such a quantum bicriticality scenario in an itinerant system is the Laves-phase $\mathrm{NbFe}_{2}$, where competing AFM and FM orders have been observed near the quantum critical point [20].

\section{COMPARISON TO $\mathrm{YbNi}_{4} \mathrm{P}_{2}$}

Our success in modeling the finite-temperature susceptibilities of $\mathrm{Yb}\left(\mathrm{Rh}_{0.73} \mathrm{Co}_{0.27}\right)_{2} \mathrm{Si}_{2}$ suggests we should look at other materials with similar phenomenology. Remarkably, the heavy-fermion ferromagnet $\mathrm{YbNi}_{4} \mathrm{P}_{2}$ has been recently found
$[10,11]$ to display a switch of magnetic response anisotropy similar to $\mathrm{Yb}\left(\mathrm{Rh}_{0.73} \mathrm{Co}_{0.27}\right)_{2} \mathrm{Si}_{2}$.

$\mathrm{YbNi}_{4} \mathrm{P}_{2}$ is a tetragonal metal with a Curie temperature of $T_{\mathrm{C}}=0.15 \mathrm{~K}$. The low-temperature ferromagnetic moment points perpendicular to the $c$ axis, as indicated by the divergent susceptibility $\chi_{a b}$ at $T_{\mathrm{C}}$. Notably, $\chi_{c}(T)$ is larger than $\chi_{a b}(T)$ for essentially all temperatures above $T_{\mathrm{C}}$. Hence, the $g$ factors obey $g_{c}>g_{a b}$, such that both the exchange and $g$-factor anisotropies appear opposite to those of $\mathrm{Yb}\left(\mathrm{Rh}_{0.73} \mathrm{Co}_{0.27}\right)_{2} \mathrm{Si}_{2}$. In $\mathrm{YbNi}_{4} \mathrm{P}_{2}$, these anisotropies lead to a crossing of $\chi_{a b}(T)$ and $\chi_{c}(T)$ at $T_{\mathrm{X}} / T_{\mathrm{C}} \approx 1.13$.

There is, however, an additional ingredient relevant for $\mathrm{YbNi}_{4} \mathrm{P}_{2}$ [in contrast to $\mathrm{Yb}\left(\mathrm{Rh}_{0.73} \mathrm{Co}_{0.27}\right)_{2} \mathrm{Si}_{2}$ ]. This material appears to be located extremely close to a quantum critical point. $T_{\mathrm{C}}$ can be suppressed with a small amount of As doping in $\mathrm{YbNi}_{4}\left(\mathrm{P}_{1-x} \mathrm{As}_{x}\right)_{2}$, with $T_{\mathrm{C}} \rightarrow 0$ extrapolated for $x \approx 0.1$. This nearly quantum critical behavior in $\mathrm{YbNi}_{4} \mathrm{P}_{2}$ is manifest in the temperature dependence of $\chi$, which follows $\chi_{c} \propto T^{-0.66}$ in the temperature range $T_{\mathrm{C}}<T<10 \mathrm{~K}$. It is this power law which cannot be reproduced by any means in our classical simulation. As seen in Fig. 2 the high-temperature behavior of $\chi$ is Curie-like, and this divergence becomes stronger upon approaching $T_{\mathrm{C}}$. In contrast, in $\mathrm{YbNi}_{4} \mathrm{P}_{2}$ the high-temperature Curie-like behavior, $\chi_{c} \propto 1 / T$, is followed by the weaker power-law divergence upon cooling.

Recalling the good match we were able to find for $\mathrm{Yb}\left(\mathrm{Rh}_{0.73} \mathrm{Co}_{0.27}\right)_{2} \mathrm{Si}_{2}$, we conclude that the latter material is dominated by effectively classical magnetism, and the quantum critical behavior of stoichiometric $\mathrm{YbRh}_{2} \mathrm{Si}_{2}$ does not appear to extend to up to $27 \%$ of Co doping.

\section{SUMMARY}

Motivated by the doping and temperature evolution of magnetism of $\mathrm{Yb}\left(\mathrm{Rh}_{1-x} \mathrm{Co}_{x}\right)_{2} \mathrm{Si}_{2}$, we studied Heisenberg models with competing anisotropies in the exchange interactions and $g$ factors. Such models possess ordered phases with spins aligned either along the $c$ axis or in the $a b$ plane, separated from each at low temperature by a first-order line which ends at a bicritical point. The proximity to this bicritical point, combined with the competition between ferromagnetic and antiferromagnetic ordering, naturally explains the "switch" in the anisotropy in the magnetic susceptibilities, as experimentally observed in $\mathrm{Yb}\left(\mathrm{Rh}_{0.73} \mathrm{Co}_{0.27}\right)_{2} \mathrm{Si}_{2}$.

While our classical local-moment modeling gives a good account of the susceptibility data of $\mathrm{Yb}\left(\mathrm{Rh}_{0.73} \mathrm{Co}_{0.27}\right)_{2} \mathrm{Si}_{2}$ near its finite-temperature phase transition, it fails to describe $\mathrm{YbNi}_{4} \mathrm{P}_{2}$, where a similar switch of response anisotropies has been observed. This highlights the importance of quantum fluctuations, as $\mathrm{YbNi}_{4} \mathrm{P}_{2}$ is located very close to a putative ferromagnetic quantum critical point.

More broadly, our results point towards bicritical behavior being relevant in certain Yb-based heavy-fermion compounds. Metallic quantum bicritical points, in particular involving phenomena with different critical exponents, are thus an exciting field for future research.

\section{ACKNOWLEDGMENTS}

We are grateful to S. Lausberg for providing experimental data and for discussions. E.C.A., moreover, thanks 

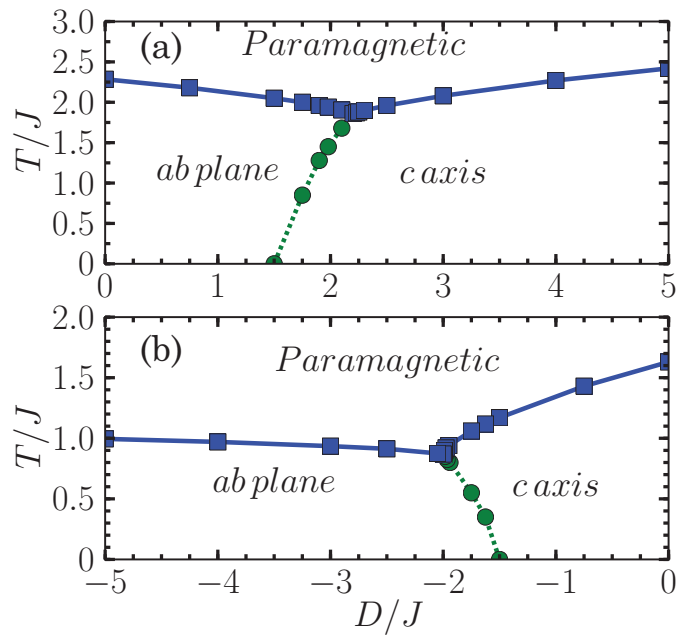

FIG. 4. (Color online) Phase diagram of model (A1) for fixed values of the exchange anisotropy: $\Delta=0.5$ in (a) and $\Delta=-0.5$ in (b). The squares represent second-order phase transitions and the circles first-order phase transitions between the two ordered states. The first order line ends at a bicritical point located at $D=2.220$ (2) (a) and $D=-1.995(5)$ (b).

F. A. Garcia for discussions. This research was supported by the DFG through FOR 960 and GRK 1621. E.C.A. was also partially supported by FAPESP. We also acknowledge ZIH at TU Dresden for the allocation of computing resources.

\section{APPENDIX: MODEL WITH SINGLE-ION ANISOTROPY}

To further explore the physics of bicriticality in a localmoment model, it is instructive to consider, in addition to the exchange anisotropy, a single-ion anisotropy of the form $-D \sum_{i}\left(S_{i}^{z}\right)^{2}$. While such a term is absent from a spin- $1 / 2$ model, it is generically present for spins $S \geqslant 1$ in a tetragonal environment.

For illustration, we consider a model with ferromagnetic nearest-neighbor exchange only, with the Hamiltonian

$$
\mathcal{H}=-\sum_{\langle i, j\rangle} \sum_{\alpha} J^{\alpha} S_{i}^{\alpha} S_{j}^{\alpha}-D \sum_{i}\left(S_{i}^{z}\right)^{2},
$$

where $D>0(D<0)$ favors spin alignment along the $c$ axis (in the $a b$ plane). For the exchange anisotropy, we stick to the parametrization $J^{x}=J^{y}=J(1+\Delta)$ and $J^{z} \equiv J$. Studies of the model (A1) have appeared before in the literature [21,22], and we complement these results here.

\section{Phase diagram}

The competition between the single-ion anisotropy $D$ and the exchange anisotropy $\Delta$ in Eq. (A1) leads to phase diagrams as shown in Fig. 4. (Figure 4(b) matches within error bars the corresponding result in Ref. [21].) At $T=0$ there is a first-order phase transition between in-plane and $c$-axis order at $D=z J \Delta / 2$, where $z$ is the lattice coordination number. This transition continues to finite $T$ and terminates at at bicritical point [22-24]. To efficiently sample all spin configurations close to this first-order phase transition, we employ the parallel-tempering algorithm [25,26]. The thermal transition at the bicritical point is in the $3 d$ Heisenberg universality class, i.e., the $\mathrm{O}(3)$ symmetry is restored here. We notice that when crossing the first-order line upon heating up the system, we always favor the state which is the ground state for $D=0$, which has a higher entropy because both spin angles are unlocked.

\section{Susceptibilities}

We now turn to the behavior of the anisotropic magnetic susceptibilities (Fig. 5). Motivated by the experimental results to $\mathrm{Yb}\left(\mathrm{Rh}_{0.73} \mathrm{Co}_{0.27}\right)_{2} \mathrm{Si}_{2}$, we only consider magnetic order
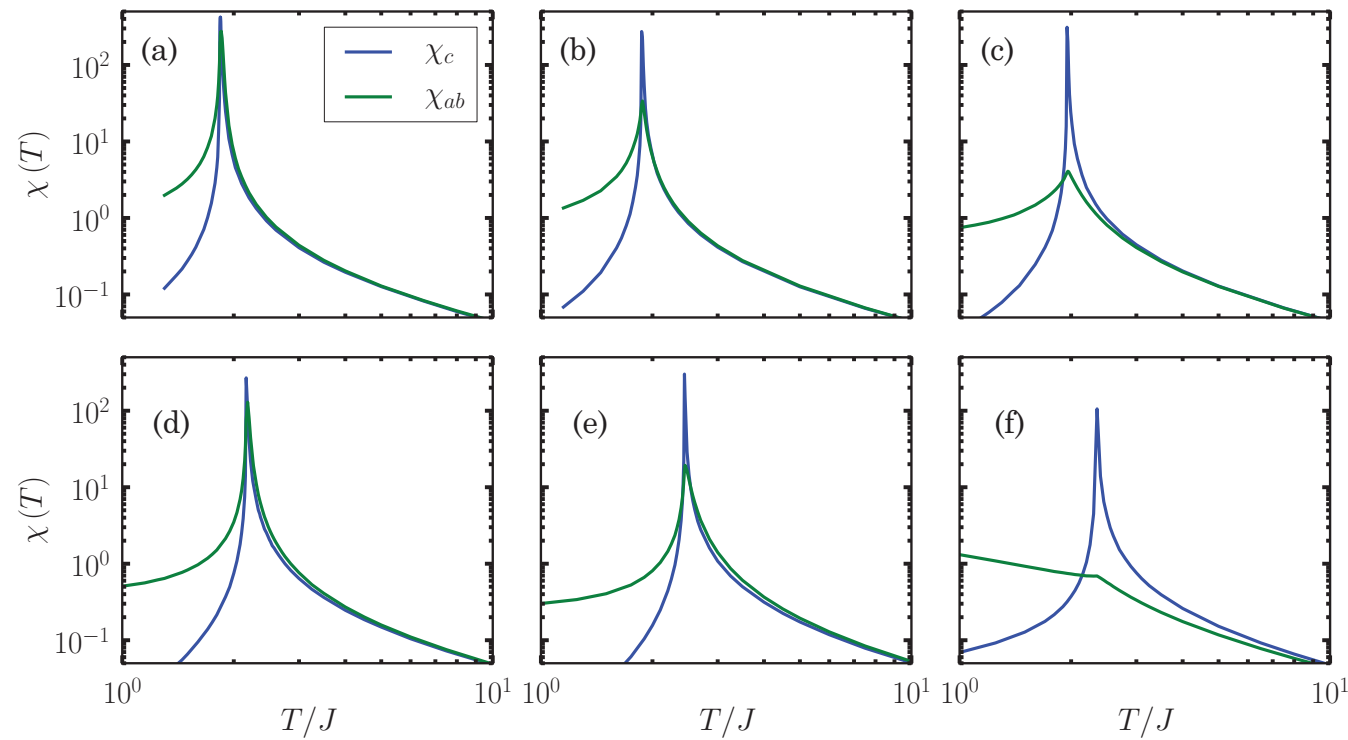

FIG. 5. (Color online) Anisotropic susceptibilities $\chi_{a b}$ and $\chi_{c}$ on a $\log -\log$ scale for $g_{a b}=g_{c}$ and $L=32$ : (a) $\Delta=0.5$ and $D=2.20 J$; (b) $\Delta=0.5$ and $D=2.25 \mathrm{~J}$; (c) $\Delta=0.5$ and $D=2.50 \mathrm{~J}$; (d) $\Delta=1.0$ and $D=4.40 \mathrm{~J}$; (e) $\Delta=1.5$ and $D=6.60 \mathrm{~J}$; and (f) $\Delta=-0.5$ and $D=-1.50 \mathrm{~J}$. 
along the $c$ axis. For all results in Fig. 5, we consider $g_{a b}=g_{c}$ and the effects of the single-ion anisotropy are encoded by the parameter $D$. In Figs. 5(a)-5(c) we see that as we approach the bicritical point, the fluctuations in $\chi_{a b}$ are enhanced. Interestingly, in 5(a) and 5(b), we even have $\chi_{a b}>\chi_{c}$ at intermediary temperatures above $T_{\mathrm{C}}$, with the two curves crossing only very close to the transition. This difference between $\chi_{a b}$ and $\chi_{c}$ is further enhanced if we increase the single-ion anisotropy $D$ [Figs. 5(d) and 5(e)]. (In both of them we keep the ratio $D / \Delta$ the same as in Fig. 5(a) so we do not move too far away from the bicritical point.) Finally, in Fig. 5(f) we consider both $D$ and $\Delta$ negative and we always have $\chi_{c}>\chi_{a b}$ for $T>T_{\mathrm{C}}$.

Therefore, we see that model (A1) naturally describes the crossing of the susceptibilities above $T_{\mathrm{C}}$ as a result of the proximity to a bicritical point. While it is true that the splitting between $\chi_{a b}$ and $\chi_{c}$ above the crossing point is small, it is still remarkable that a purely local-moment description is able to capture such crossover, an observation which motivates us to associate this crossing to the proximity of a bicritical point.
[1] P. Gegenwart, Q. Si, and F. Steglich, Nat. Phys. 4, 186 (2008).

[2] H. von Löhneysen, A. Rosch, M. Vojta, and P. Wölfle, Rev. Mod. Phys. 79, 1015 (2007).

[3] O. Trovarelli, C. Geibel, S. Mederle, C. Langhammer, F. M. Grosche, P. Gegenwart, M. Lang, G. Sparn, and F. Steglich, Phys. Rev. Lett. 85, 626 (2000).

[4] P. Gegenwart, J. Custers, C. Geibel, K. Neumaier, T. Tayama, K. Tenya, O. Trovarelli, and F. Steglich, Phys. Rev. Lett. 89, 056402 (2002).

[5] S. Friedemann, T. Westerkamp, M. Brando, N. Oeschler, S. Wirth, P. Gegenwart, C. Krellner, C. Geibel, and F. Steglich, Nat. Phys. 5, 465 (2009).

[6] C. Klingner, C. Krellner, M. Brando, C. Geibel, F. Steglich, D. V. Vyalikh, K. Kummer, S. Danzenbächer, S. L. Molodtsov, C. Laubschat, T. Kinoshita, Y. Kato, and T. Muro, Phys. Rev. B 83, 144405 (2011).

[7] P. Gegenwart, J. Custers, Y. Tokiwa, C. Geibel, and F. Steglich, Phys. Rev. Lett. 94, 076402 (2005).

[8] S. Lausberg, A. Hannaske, A. Steppke, L. Steinke, T. Gruner, L. Pedrero, C. Krellner, C. Klingner, M. Brando, C. Geibel, and F. Steglich, Phys. Rev. Lett. 110, 256402 (2013).

[9] C. Klingner, C. Krellner, M. Brando, C. Geibel, and F. Steglich, New J. Phys. 13, 083024 (2011).

[10] C. Krellner, S. Lausberg, A. Steppke, M. Brando, L. Pedrero, H. Pfau, S. Tence, H. Rosner, F. Steglich, and C. Geibel, New J. Phys. 13, 103014 (2011).

[11] A. Steppke, R. Küchler, S. Lausberg, E. Lengyel, L. Steinke, R. Borth, T. Lühmann, C. Krellner, M. Nicklas, C. Geibel, F. Steglich, and M. Brando, Science 339, 933 (2013).
[12] J. Sichelschmidt, V. A. Ivanshin, J. Ferstl, C. Geibel, and F. Steglich, Phys. Rev. Lett. 91, 156401 (2003).

[13] D. P. Landau and K. Binder, A Guide to Monte Carlo Simulations in Statistical Physics, 3rd ed. (Cambridge University Press, Cambridge, 2009).

[14] A nice example is the itinerant helimagnet $\mathrm{MnSi}$, which can be well described using local-moment models, as shown in S. Buhrandt and L. Fritz, Phys. Rev. B 88, 195137 (2013).

[15] T. Gruner, J. Sichelschmidt, C. Klingner, C. Krellner, C. Geibel, and F. Steglich, Phys. Rev. B 85, 035119 (2012).

[16] C. L. Henley, Phys. Rev. Lett. 62, 2056 (1989).

[17] J. A. Hertz, Phys. Rev. B 14, 1165 (1976).

[18] T. Meng, A. Rosch, and M. Garst, Phys. Rev. B 86, 125107 (2012).

[19] In layered metals, quantum (bi)criticality may be accompanied by dimensional crossover phenomena: M. Garst, L. Fritz, A. Rosch, and M. Vojta, Phys. Rev. B 78, 235118 (2008).

[20] D. Moroni-Klementowicz, M. Brando, C. Albrecht, W. J. Duncan, F. M. Grosche, D. Grüner, and G. Kreiner, Phys. Rev. B 79, 224410 (2009).

[21] R. T. S. Freire, J. A. Plascak, and B. V. d. Costa, Braz. J. Phys. 34, 452 (2004).

[22] W. Selke, Phys. Rev. E 87, 014101 (2013).

[23] Y. Shapira and S. Foner, Phys. Rev. B 1, 3083 (1970).

[24] W. Selke, Phys. Rev. E 83, 042102 (2011).

[25] K. Hukushima and K. Nemoto, J. Phys. Soc. Jpn. 65, 1604 (1996).

[26] C. E. Fiore, Phys. Rev. E 78, 041109 (2008). 